\title{
Migrantes marginalizadas y sus reivindicaciones de justicia global
}

Marginalized migrants and their demands for global justice

ZuZANA Uhde

Czech Academy of Sciences, Institute of Sociology zuzana.uhde@soc.cas.cz

Orcid: 0000-0002-9885-4260

DOI: http://dx.doi.org/10.15366/bp.2020.23.004 Bajo Palabra. II Época. No23. Pgs: 103-130 


\begin{abstract}
Este trabajo ha sido financiado por el proyecto de investigación Global Conflicts and Local Interactions
(Strategy AV21) y el apoyo institucional de la Academia Checa de Ciencias (RVO: 68378025). Este texto ha sido traducido desde el inglés por MariaCaterina La Barbera y Juan Carlos Velasco.
\end{abstract}

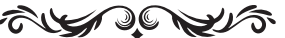

\section{Resumen}

Las prácticas de migración transnacional representan un desafío para las ciencias sociales y la articulación teórica de la justicia global. Este artículo sitúan las prácticas de migración transnacional en el contexto de una economía geopolítica de globalización capitalista y se argumenta la necesidad de abandonar el nacionalismo metodológico como marco analítico definitorio. Desde esta perspectiva, se arguye que, si bien las personas migrantes marginalizadas no comparten necesariamente una conciencia cosmopolita, estos pueden definirse como actores de los procesos de cosmopolitismo realmente existente si se les concibe como un grupo estructural. Las luchas cotidianas de los migrantes son sólo aparentemente individuales. La teoría social crítica cosmopolita permite referirse a la crítica vivida por los migrantes como una fuente de reivindicaciones generalizables de justicia global, aunque no adopte la forma de la protesta política tradicional. A continuación, se examina la dimensión de género de las reivindicaciones de justicia global mediante el análisis de las reivindicaciones de cuidados derivadas de la crítica vivida de las trabajadoras domésticas migrantes, que implican demandas más ambiciosas de justicia global que las avanzadas por los colectivos organizados.

Palabras clave: teoría social crítica cosmopolita, migración transnacional, justicia global, critica vivida, cuidados.

\section{Abstract}

Transnational migration practices represent a challenge for social sciences and theoretical articulation of global justice. This article locates the transnational migration practices in a context of a geopolitical economy of capitalist globalization and argues for a necessary shift away from methodological nationalism as a defining analytical framework. Following this, the author presents the argument that while marginalized migrants do not necessarily share a cosmopolitan consciousness, they can be defined as actors of really-existing processes of cosmopolitanism if conceptualized as a structural group. She argues that while migrants' everyday struggles are seemingly only individual. Cosmopolitan critical social theory allows to refer to a migrants' lived critique as a source of generalizable claims for global justice although it does not take the form of traditional political protest. She then discusses the gendered dimension of the claims for global justice through analysis of care claims derived from migrant domestic workers' lived critique which implies a more ambitious demands on global justice than those asserted by organized collectivities.

Keywords: cosmopolitan critical social theory, transnational migration, global justice, lived critique, care. 


\section{Introducción}

LAS PRÁCTICAS DE MIGRACIÓN TRANSNACIONAL representan un desafío para las ciencias sociales y la articulación teórica de la justicia global. Las pautas contemporáneas de migración transnacional no pueden entenderse plenamente en el marco de las relaciones entre los estados. Si bien las personas se desplazan a través de las fronteras de los estados nacionales, su movilidad forma parte de dinámicas globales económicas, políticas y culturales. Las actuales tendencias nacionalistas, la externalización y la subcontratación del control fronterizo así como el consiguiente deterioro del régimen de derechos humanos de las personas migrantes se interpretan falsamente como una confirmación de la relevancia de un marco analítico definido por el estado-nación. No obstante, esas tendencias también responden a interacciones globales y prácticas transnacionales. Las actuales tendencias nacionalistas son un intento de abordar los problemas utilizando medios caducos que, en verdad, no pueden ofrecer una solución. Sin embargo, el grueso de la investigación social sobre las migraciones sigue funcionando con el marco analítico del nacionalismo metodológico. Al hacerlo, legitiman medidas políticas que, en el mejor de los casos, se limitan a funcionar para unos pocos migrantes selectos, dejando intacto un arreglo geopolítico global profundamente injusto. Además, si se consideran los procesos sociales desde esta perspectiva, la crítica vivida (lived critique) de los grupos marginalizados en un contexto transnacional -y sus legítimas reivindicaciones de justicia global- quedan inevitablemente fuera del foco de la investigación.

En este artículo presento, en primer lugar, mi marco de referencia empleando la literatura sobre migraciones transnacionales desde la perspectiva de la teoría social crítica cosmopolita con el fin de articular una crítica del nacionalismo metodológico y situar las prácticas de migración transnacional dentro de los procesos realmente existentes de cosmopolitismo. En la segunda parte, sitúo las prácticas de migración transnacional en una economía geopolítica de la globalización capitalista y argumento que, si bien los migrantes marginalizadas no comparten necesariamente una conciencia cosmopolita, si se les conceptualiza como un grupo estructural pueden definirse como actores de los procesos de cosmopolitismo realmente existente. A continuación, elaboro el concepto de crítica vivida e identifico el reconocimiento erróneo estructural (structural misrecognition) que experimentan las personas migrantes transnacionales. En la parte final, examino la dimensión de género de las 
reivindicaciones de justicia global mediante el análisis de las reivindicaciones de cuidados que derivan de la crítica vivida de las trabajadoras domésticas migrantes.

\section{Límites del nacionalismo metodológico}

LAS INTERACCIONES GLOBALES no sólo intensifican la migración y los conflictos, sino que también crean posibilidades para que los agentes amplíen el alcance de sus luchas más allá de las fronteras de los estados nacionales. Sin embargo, el análisis de las injusticias estructurales transnacionales y globales, así como la formulación de respuestas a las mismas, requieren una revisión de la visión actualmente dominante, basada exclusivamente en la división en estados nacionales. Esto se denomina nacionalismo metodológico. El nacionalismo metodológico refleja, y a la vez legitima, una práctica igualmente inadecuada de la política real, que identifica la responsabilidad de la justicia global dentro de las fronteras de los estados-nación. Aunque el nacionalismo metodológico se presenta como un enfoque neutral, se basa en supuestos ideológicos ocultos vinculados a la soberanía territorial de los estados-nación y a la conceptualización de la sociedad como una unidad social que se superpone al territorio del estado-nación moderno (Beck y Sznaider 2006; Wimmer y Glick Schiller 2002). Desde el punto de vista del nacionalismo metodológico, la migración parece ser una desviación problemática de la norma (Glick Schiller 2009; Wimmer y Glick Schiller 2002; Sager 2018; Castles et al. 2015).

En las últimas décadas, las investigaciones sobre las migraciones internacionales han aumentado y el campo de estudio se ha desarrollado considerablemente, integrando diferentes perspectivas teóricas y enfoques disciplinarios (p. ej., Brettell y Hollifield 2000, King 2013). Sin embargo, Stephan Castles (2010) ha sostenido que los actuales estudios sobre migración se enfrentan a varias deficiencias institucionalizadas que contribuyen a la legitimación de una política real de la migración sesgada: fragmentación disciplinaria y desconexión de la teoría social contemporánea de la globalización, una orientación y un enfoque político centrado en los países receptores, la separación de la migración forzada (p.ej., refugiados políticos, conflictos bélicos, ambientales) de la migración económica (migración laboral), y otros tipos de fragmentación temática o geográfica, así como un marco analítico conformado por el nacionalismo metodológico nunca cuestionado (Castles 2010). Alex Sager (2018), por su parte, sostiene que las teorías políticas (específicamente la tradición angloamericana) también han adoptado mayoritariamente este mismo sesgo cognitivo y, en consecuencia, el enfoque predominante se centra en la inmigración. Categorías como ciudadano, inmigrante, emigrante, turista o "fuga 
de cerebros" respectivamente están incrustadas en el paradigma del nacionalismo metodológico sin que tenga reflejo en las teorías políticas.

Los estudios que han hecho avanzar una crítica del nacionalismo metodológico son cada vez más influyentes en la elaboración de un nuevo marco conceptual y teórico para analizar las migraciones transnacionales en el contexto de la economía globalizada. Van más allá de concebir a las personas migrantes como una amenaza o anormalidad y sitúan la migración entre otros procesos sociales e interacciones transfronterizas en relación con las instituciones sociales, las formas de vida dentro y fuera de las fronteras, las desigualdades y los conflictos globales (Glick Schiller 2009; Vertovec 2009). Sin embargo, varios estudios sostienen que poner el foco de forma predominante en las formas sociales y la formación de la identidad existentes (p.ej., las remesas, las redes y relaciones sociales transnacionales, las diásporas transnacionales) limita sus posibilidades analíticas. Por esta razón, se requiere una conexión teórica más estrecha con el sistema económico global y la geopolítica de los conflictos y jerarquías de poder (Castles 2010; Castles et al. 2015; Glick Schiller 2009).

Aunque los estudios sobre migraciones entienden en ocasiones el transnacionalismo de manera simplista como un estudio de los continuos vínculos de las personas migrantes entre aquí y allá y sus múltiples pertenencias (Waldinger y Fitzgerald 2004), o como un enfoque unilateral orientado a los actores, sugiero que es más fructífero considerar una perspectiva analítica que supera el supuesto normativo oculto del nacionalismo metodológico, que concibe el estado-nación como el contenedor de todos los procesos sociales en los que deben encajar los migrantes. Así pues, el término transnacional se refiere a las escalas donde se encuentran las causas y consecuencias de las prácticas y formas sociales, más que a los lugares donde se producen. El esfuerzo analítico se concentra en la comprensión del nexo entre la migración transnacional y las relaciones cambiantes entre los agentes estatales y no estatales dentro del capitalismo global. Por una parte, esta comprensión de las prácticas de migración transnacional permite concebir la dinámica de la migración más allá de la categoría del estado-nación como unidad fundamental de análisis. Por otra parte, esta comprensión no postula que todo migrante lleve una vida transnacional, sino que permite captar la relación entre diversos niveles socio-espaciales.

Las ciencias sociales construidas sobre un nacionalismo metodológico aparentemente neutral distorsionan la producción de conocimientos y no proporcionan un marco adecuado para explorar los procesos sociales contemporáneos, incluyendo las migraciones (Beck 2006; Wimmer y Glick Schiller 2002). El marco y la escala del análisis determinan tanto el objeto del análisis como los contextos que se consi- 
derarán. Esta es una objeción fundamental al nacionalismo metodológico: aunque determinados procesos pueden investigarse dentro de un estado, la aplicación del nacionalismo metodológico predetermina el tema y el contexto del análisis en la medida en que omite las posibles causas y consecuencias de los fenómenos y procesos individuales que van más allá de las fronteras del estado-nación. El nacionalismo metodológico distorsiona la producción de conocimientos de dos maneras. En primer lugar, incluso los procesos que se producen espacialmente dentro de un estado no pueden ser captados analíticamente dentro del marco de referencia del estado-nación porque sus raíces y conexiones transnacionales permanecen fuera del foco de investigación. En segundo lugar, la aplicación de la perspectiva del nacionalismo metodológico distorsiona el significado de los procesos y fenómenos específicos porque sólo se consideran los que ocurren dentro del estado-nación y luego se comparan a nivel internacional; es decir, las unidades de análisis siguen siendo estados-nación.

Ulrich Beck, un destacado defensor del paradigma cosmopolita en las ciencias sociales, sostiene que dichas ciencias deben centrarse en los procesos de cosmopolitización realmente existentes como primer paso necesario para cualquier propuesta normativa. En su teoría crítica, Beck desarrolla la posición del realismo cosmopolita centrándose en las tendencias cosmopolitas emergentes en las formas de vida, normas e instituciones transnacionales (Beck 2006; Beck y Sznaider 2006). Análogamente, Robert Fine sostiene que el cosmopolitismo no es una idea preconcebida, sino más bien un programa de investigación que desarrolla una perspectiva para abordar la realidad social existente -que él llama la era del cosmopolitismo- dónde las posibilidades de un futuro cosmopolita están abiertas aunque no se hayan desarrollado plenamente hasta la fecha (Fine 2007). Para superar las deficiencias de las principales ciencias sociales, Beck propone la perspectiva analítica del cosmopolitismo metodológico (Beck 2006; Beck y Sznaider 2006). En las ciencias sociales, esta perspectiva apunta a las interacciones globales que dan lugar a nuevas formas de sociabilidad, la transformación del papel de los estados-nación así como las prácticas económicas, políticas y culturales transnacionales, sus consecuencias no deseadas y los riesgos asociados. Sin embargo, desde la perspectiva del cosmopolitismo metodológico, también se puede examinar la dinámica local y nacional en su complejidad social y en una relación dialéctica con la realidad social cosmopolita en evolución. Es decir, el cosmopolitismo metodológico no predetermina el objeto de la investigación, sino los puntos de partida analíticos a partir de los cuales se explora una cuestión particular.

Siguiendo a Beck y a Fine, distingo entre la teoría social crítica cosmopolita, que se basa en fundamentos intersubjetivos y en el análisis contextualizado histórica- 
mente, y la teoría liberal cosmopolita, que se basa en fundamentos demasiado individualistas y en abstracciones y prescripciones políticas ahistóricas ${ }^{1}$. La teoría social crítica cosmopolita formula, por el contrario, argumentos normativos sobre la base del análisis de los procesos de cosmopolitización realmente existentes y las reivindicaciones de los actores sociales. Los momentos críticos, descriptivos y normativos no están separados, sino que se integran en un enfoque conceptual que combina una concepción universal de la humanidad con solidaridades particulares y vínculos locales (Beck 2006; Delanty 2006; Fine 2007; Sager 2018; Turégano 2019)². El énfasis en los procesos de cosmopolitización realmente existentes hace que la teoría social crítica cosmopolita haga de contrapunto a la teoría política cosmopolita normativa unilateral con la que a menudo se equipara erróneamente el término cosmopolitismo (Estévez 2014; Kang 2014).

Las prácticas de migración transnacional son una manifestación de la transnacionalización de las formas de vida y las prácticas sociales que son una condición previa para el surgimiento de la conciencia cosmopolita. Sin embargo, la transnacionalización de las prácticas sociales, que tiene un significado crítico o descriptivo, no es lo mismo que el cosmopolitismo, y puede no conducir necesariamente al surgimiento de una conciencia cosmopolita. El cosmopolitismo se refiere a una crítica reflexiva y al horizonte normativo de las ideas sobre una sociedad alternativa y las demandas de justicia de los actores sociales. Este punto es subrayado por Gerard Delanty (2006), quien afirma que una imaginación cosmopolita es más que la transnacionalización y la pluralización de las formas de vida. Es una apertura reflexiva e internalizada al mundo, resultado de un proceso hermenéutico y cognitivo de aprendizaje "en el que las codificaciones tanto del Yo como del Otro se transforman" (Delanty 2006: 37). Beck explica que cuando habla de los procesos de cosmopolitización realmente existentes, él excluye lo que denomina el falso cosmopolitismo de una clase capitalista transnacional y de las elites globales, que se limitan a instrumentalizar los argumentos cosmopolitas para reproducir y consolidar a escala global los actuales arreglos geopolíticos y económicos.

Para Beck, los actores de los procesos de cosmopolitización realmente existentes son los actores de la subpolítica global, así como los migrantes y los miembros de

\footnotetext{
${ }^{1}$ Para un análisis de las diferentes interpretaciones del cosmopolitismo en la teoría política, véase Ingram (2013).

${ }^{2}$ Marek Hrubec sostiene que para poder proporcionar criterios normativos que se basen en las reflexiones críticas de los actores sobre la injusticia y sus reivindicaciones normativas, la teoría crítica debe basarse en tres elementos fundamentales articulados en sus relaciones mutuas: crítica, explicación y normatividad (Hrubec 2012). El equilibrio de estos tres elementos le permite conceptualizar los criterios normativos incorporados en el desarrollo histórico de las luchas sociales y analizar las tendencias normativas positivas articuladas en el pasado y la facticidad actual, especialmente en las interacciones y conflictos transnacionales y globales.
} 
sus familias transnacionales ${ }^{3}$. Beck sostiene que los procesos de cosmopolitización realmente existentes son las consecuencias imprevistas de la radicalización de la modernidad que conlleva tendencias autodestructivas que se manifiestan en forma de riesgos globales. Sostiene que la percepción de los riesgos globales por parte de los actores, y en cierta medida las elecciones forzadas en respuesta a ellos, crean el cosmopolitismo cotidiano (Beck 2006 2009; Beck y Beck-Gernsheim 2013). Como tal, los procesos de cosmopolitismo realmente existentes no conducen necesariamente a un acuerdo cosmopolita positivo. Al mismo tiempo, no todas las reivindicaciones y expectativas de los actores marginalizados están justificadas si implican una limitación de los derechos de otros grupos. Para hacer esta distinción y comprender los procesos de cosmopolitización realmente existentes, necesitamos una articulación teórica de los criterios normativos para la crítica. Sin embargo, tales procesos de cosmopolitización abren un espacio de posibilidades en el que podemos identificar las fuentes de la crítica cosmopolita y las reivindicaciones de los actores por la justicia global, aunque no internalizan necesariamente una conciencia cosmopolita. La crítica vivida de los grupos marginalizados en un contexto transnacional presenta un vector que debe orientar nuestra teorización de la justicia global.

\section{Crítica vivida de las personas migrantes al capitalismo global}

LA COMPRENSIÓN DE LA MIGRACIÓN TRANSNACIONAL en el contexto de la economía política global plantea problemas de la estricta distinción entre los migrantes políticos en calidad de refugiados (que merecen ser admitidos y recibir protección jurídica) y los denominados migrantes económicos (que no merecen ser admitidos). Esta distinción, en sí misma, se basa en una idea naturalizada de que el estado-nación es el principal actor de la política global. También supone falsamente que mientras el primer grupo no tiene otra posibilidad que migrar, el otro grupo decide libremente abandonar su país de origen. Aunque la migración es una decisión activa en las estrategias adaptativas de los migrantes, no es una elección libre de limitaciones estructurales. La vida en la migración es una continuación de su lucha contra la injusticia global. Este enfoque despolitizado de la migración económica también pasa por alto la integración de los factores económicos y sociales en la

\footnotetext{
3 Beck no desarrolla sistemáticamente esta noción inclusiva de los actores. Habla de subpolítica cuando se refiere a los actores políticos que están fuera de las instituciones políticas tradicionales; sin embargo, reserva la subpolítica principalmente para los colectivos organizados (Beck 2009). Por lo tanto, parece que los actores marginalizados escapan a su atención a menos que se organicen colectivamente.
} 
economía global: los problemas locales están relacionados con las prácticas transnacionales tanto de manera directa como de forma no intencional. Raúl Delgado Wise (2018: 750-751) amplía el concepto de migración forzosa más allá del entendimiento convencional al referirse al grupo de refugiados, solicitantes de asilo y personas desplazadas, y sostiene que la dinámica del capitalismo global produce condiciones estructurales en las que "la migración se ha convertido esencialmente en un desplazamiento forzado de población". Específicamente, la migración forzosa incluye la migración debida a violencia, conflicto y catástrofe, tráfico de personas, despojo, exclusión y desempleo, y deportaciones. La llamada migración económica es una forma de migración forzosa, aunque no todas las personas migrantes son migrantes forzosos. Cuando hablo de migrantes transnacionales, me refiero específicamente a los grupos marginalizados de migrantes que se ven obligados a marcharse en diversos grados por diferentes razones (conflictos, cambios ambientales, luchas económicas, delitos, etc.). La distinción fundamental es entonces entre las personas que cruzan las fronteras debido a diferentes tipos de conflictos o dificultades y las personas que se encuentran en una posición ventajosa debido a sus aptitudes y su capital económico, social o político y que tienen acceso a un régimen de movilidad transnacional mucho más flexible.

En general, los análisis de las prácticas transnacionales del capitalismo global muestran que resultan en un aumento de las desigualdades y la pobreza, conflictos y guerras transnacionales, deterioro de los servicios públicos y la gobernanza, y mayores riesgos globales, incluidos los medioambientales (Robinson 2014; Sklair 2003). Estos factores son co-creadores de las causas estructurales de las migraciones transnacionales. No obstante, aunque las causas estructurales de la migración sean transnacionales y globales, la respuesta política a la migración está vinculada al sesgo del estado-nación. Leslie Sklair (2002) sostiene que es importante hacer una distinción entre la globalización genérica y la globalización capitalista. La globalización capitalista representa una forma histórica particular de globalización. Sin embargo, para hacer frente a las injusticias estructurales globales creadas por el capitalismo global se necesitan algunas interacciones globales conectadas con la globalización genérica.

La economía política crítica ofrece importantes perspectivas. Pone de relieve el contexto económico, cultural y geopolítico de la globalización capitalista que está dando forma a las actuales prácticas de migración transnacional. William Robinson $(2004 ; 2014)$ pone en tela de juicio la comprensión de una economía global como una competencia de capitales nacionales en la arena internacional y formula una teoría del capitalismo global. Interpreta el desarrollo contemporáneo como un cambio cualitativo acompañado de la disrupción de los circuitos de acumula- 
ción nacionales y su reintegración en uno nuevo, caracterizado por un sistema de producción y financiero global. En otras palabras, sostiene que el sistema-mundo capitalista se transformó en el sistema capitalista global. Fundamenta su análisis destacando la creciente concentración y control del capital en mano de grandes corporaciones transnacionales por parte de una clase capitalista transnacional que no tiene ningún "interés nacional" particular (Robinson 2014). Sostiene que, si bien la fase anterior del desarrollo capitalista se caracterizó por una ampliación extensiva y la inclusión de las macrorregiones en un sistema económico global, una estrategia esencial de acumulación de beneficios en la economía transnacional y global se ha centrado en la ampliación intensiva del capitalismo. Esta ampliación intensiva se caracteriza por la mercantilización de áreas y prácticas de la vida social que antes estaban excluidas de las relaciones de mercado (Robinson 2004). El estado ya no es el principio organizador fundamental, pero sigue siendo el poder ejecutivo del capitalismo global ${ }^{4}$. También muestra que, detrás de las estadísticas de los estados-nación individuales, se oculta una tendencia en la que una división internacional del trabajo se está transformando en "una división global del trabajo en la que las actividades productivas centrales y periféricas están dispersas tanto dentro como entre los países" (Robinson 2014: 39). Así, por ejemplo, los migrantes transnacionales se incluyen en un mercado laboral globalizado como mano de obra móvil y barata. Esta explotación estructural redunda en los intereses más amplios del capital y es posible gracias a la "ilegalidad" y la "deportabilidad" producidas por el estado (De Genova 2002). El antropólogo social Nicholas De Genova (2018) sostiene que el espectáculo fronterizo de la exclusión (principalmente de los llamados migrantes económicos) sólo enmascara el papel productivo de las fronteras que producen migrantes "ilegales" subordinados que son explotados como mano de obra barata y desechable (deportable), lo que es a la vez indispensable en muchos sectores del mercado.

Sugiero que la crítica vivida de los migrantes pone en tela de juicio la legitimidad del capitalismo global y del marco institucional y jurídico definido por el estado-nación. Para elaborar el concepto de crítica vivida me baso en la teoría crítica y en su premisa fundamental de que el análisis social debe basarse en experiencias de

\footnotetext{
${ }^{4}$ La teórica crítica Leslie Sklair (2003), que redefinió la definición clásica de clase capitalista basada en la propiedad de los medios de producción para incluir otras formas de capital además de las económicas, es decir, las políticas, el conocimiento y las culturales. Según Sklair, la clase capitalista transnacional incluye hoy en día no sólo a los propietarios de las grandes corporaciones y a sus directivos (la fracción empresarial), sino también a los políticos y burócratas globalizadores a nivel internacional, nacional y local que se alinean con el capital global (la fracción estatal), a los profesionales del mercado laboral global (la fracción técnica) y a los actores que controlan los medios de comunicación (la fracción consumista) (Sklair 2003: 17-23). Esta es la clave para comprender la relación entre el estado y otros actores privados en la globalización capitalista actual.
} 
injusticia históricamente específicas (Honneth 2006; Hrubec 2012; Young 2000, 1990). Desde esta perspectiva, los criterios normativos de justicia y legitimidad no se formulan de arriba abajo, sino que se deducen de la protesta y la crítica de los actores marginalizados y oprimidos. Sus reivindicaciones normativas se articulan sobre la base de esta crítica. Sin embargo, prestar atención exclusivamente a las formas tradicionales de protesta política (manifestaciones, huelgas, campańas, etc.) significaría ignorar una parte sustancial de la protesta en la sociedad. Esas reivindicaciones prepolíticas, que se expresan en las luchas cotidianas de los actores, están en el centro de la teoría crítica del reconocimiento elaborada especialmente por Axel Honneth (2006). Iris M. Young $(2006,2011)$ y Marek Hrubec (2013) amplían el alcance de la teoría crítica con el fin de abordar las experiencias de la injusticia global. Mientras Young desarrolla el concepto de injusticia estructural global, Hrubec integra en su enfoque las luchas por el reconocimiento de los actores más marginalizados dentro de las interacciones globales, a saber, las personas desposeídas a nivel global. Sobre la base de este enfoque teórico crítico, sostengo que la comprensión de los y las migrantes marginalizados como grupo estructural permite que la teoría social crítica cosmopolita se refiera a la crítica vivida por los migrantes como fuente de reivindicaciones de justicia global, aunque no adopte la forma de la protesta política tradicional.

Las estructuras institucionalizadas del capitalismo global que provocan la migración constituyen una injusticia estructural. Iris M. Young define la injusticia estructural como

un tipo de mal moral distinto de la acción ilícita de un agente individual o de las políticas deliberadamente represivas de un estado. La injusticia estructural se produce como consecuencia de las acciones de muchas personas e instituciones en pos de sus objetivos e intereses particulares, dentro de determinadas reglas institucionales y normas aceptadas (Young 2006: 114).

Las personas actúan de acuerdo con las condiciones y recursos disponibles según su condición social, historia y contexto cultural, creando sus estrategias de vida dentro de redes de relaciones intersubjetivas bajo la restricción de estructuras externas. Su agencia individual y colectiva reproduce y también cambia las estructuras existentes, que a su vez limitan su agencia y definen sus condiciones futuras. La relación entre los individuos y las estructuras sociales se define mediante un proceso de refuerzo mutuo. El enfoque de Young nos permite descubrir las fuentes de las injusticias estructurales localizadas en las relaciones intersubjetivas sin necesidad de identificar una fuente individualizada de estas injusticias. Aplica esta comprensión de la injusticia estructural al nivel global que no puede caracterizarse como una con- 
secuencia directa o intencional de las acciones de los individuos u organizaciones, sino que surge de procesos sociales estructurales que interconectan a las personas a través de las fronteras (Young 2006, 2011). El carácter de estas injusticias da lugar a obligaciones globales de justicia y a las correspondientes responsabilidades de remediar los resultados injustos de las estructuras sociales globales. Young entiende esto como un modelo de conexión social de responsabilidad que está orientado hacia el futuro (centrado en remediar los resultados injustos), compartido y, por lo tanto, distribuido contextualmente (en contraste con la responsabilidad colectiva).

Si bien todos los integrantes del sistema de relaciones estructurales e institucionales se encuentran en circunstancias de justicia que les imponen obligaciones respecto de todos los demás, quienes están institucional y materialmente en situación de poder hacer más para cambiar las condiciones de vulnerabilidad tienen mayores obligaciones (Young 2006: 106).

Aunque la migración se tematiza a menudo en términos de interacciones interculturales, como diferencias entre las normas y prácticas culturales de las personas migrantes y las de la mayoría, o en términos de objetivos políticos conflictivos de asimilación e inclusión, estas perspectivas parecen menos importantes si uno se centra en la injusticia estructural. Según Young, las diferencias culturales se convierten en una cuestión política si están, como suelen estar, vinculadas a desigualdades estructurales. Muchas situaciones presentadas como conflictos culturales son más bien conflictos sociopolíticos porque se basan en disputas por el territorio, los recursos, la participación en el mercado laboral o el poder de decisión. Young distingue entre grupos culturales y estructurales (Young 2000). Mientras que los grupos culturales se unen por el idioma, las prácticas cotidianas, las formas de sociabilidad, las convenciones estéticas o religiosas, que ofrecen a sus integrantes ciertos medios de expresión y comunicación comunes y crean un entorno de afinidad mutua; los grupos estructurales están relacionados por aspectos materiales o psicológicos y con el estatus social. Según Young, "un grupo social estructural es un conjunto de personas que se encuentran en una posición similar en las relaciones interactivas e institucionales que condicionan sus oportunidades y perspectivas de vida" (Young 2000: 97). Entre los ejemplos de diferencias estructurales que ofrece Young figuran las relaciones constituidas en base al género, la "raza", la clase, la sexualidad y la discapacidad 5 . Con el objeto de desenmarañar las limitaciones estructurales a

\footnotetext{
5 Young propone entender a las mujeres como un grupo estructural. Según Young, el género es "una forma particular de posicionamiento social de los cuerpos vividos en relación unos con otros dentro de instituciones y procesos histórico y socialmente específicos que tienen efectos materiales en el entorno en el que las personas actúan y reproducen las relaciones de poder y privilegio entre ellas” (Young 2005: 22). Por lo tanto, el género no significa identidad sino un vínculo estructural específico entre las condiciones institucionales, las posibilidades de vida de los individuos y su realización.
} 
que se enfrentan los migrantes, es necesario entenderlos (a pesar de las diferencias entre grupos) como un grupo estructural de personas que comparten una posición específica en las estructuras sociales y las relaciones institucionalizadas que los hace vulnerables a la marginación, la exclusión y el sufrimiento material y social, lo que limita su capacidad de realizar y desarrollar sus capacidades, expresar sus opiniones o experiencias y participar en la definición de las condiciones de su vida.

La construcción de fronteras en el contexto de la globalización capitalista determina la posición estructural de las personas migrantes. Si bien las relaciones políticas globales se organizan sobre la base de una comprensión naturalizada de las fronteras de los estados-nación, las prácticas transnacionales realmente existentes que constituyen la globalización capitalista se caracterizan por procesos de fronterización duales. Los procesos de fronterización no detienen la movilidad del capital y las clases privilegiadas a nivel global. Se dirigen selectivamente contra los migrantes marginalizados como sujetos racializados. Además, la movilidad del capital requiere algunas formas de fronteras que son rentables para las prácticas económicas transnacionales y el capital global, permitiendo la explotación de la mano de obra barata de los migrantes, así como diferentes flujos financieros ilícitos transfronterizos, por ejemplo, la evasión fiscal, la facturación comercial falsa, la fuga de capitales y también los beneficios cada vez mayores de la militarización y la securitización del control fronterizo. Si bien los estados individuales crean conjuntamente las condiciones que motivan las migraciones transnacionales, en el actual mundo globalmente interconectado, y frente a poderosas instituciones y empresas transnacionales, los estados-nación no siempre pueden proteger los derechos de las personas que se encuentran en sus territorios.

En su teoría crítica del reconocimiento, Axel Honneth (2006) interpreta los procesos de emancipación como un orden de reconocimiento institucionalizado emergente. Las teorías de reconocimiento se basan generalmente en el argumento de que los individuos deben su integridad personal a las relaciones intersubjetivas con los demás. Según Honneth, el reconocimiento surge del proceso histórico de las luchas contra el reconocimiento erróneo y las reivindicaciones de reformulación de los principios normativos que rigen la sociedad o una esfera específica de la sociedad. Honneth basa su teoría del reconocimiento en una interpretación de la diferenciación histórica entre tres esferas de reconocimiento y tres formas relacionadas de relación con el yo: relaciones íntimas regidas por el principio normativo del amor, que proporciona al individuo una confianza en sí mismo básica; relaciones jurídicas guiadas por el principio de la igualdad de derechos, que proporciona el respeto de sí mismo; y el reconocimiento social que se fundamenta en el criterio del rendimiento individual como fuente de autoestima. Sin embargo, Honneth articu- 
la las reivindicaciones prepolíticas expresadas en las luchas por el reconocimiento sin tener en cuenta su dimensión global (Honneth 2006). Marek Hrubec desarrolla la perspectiva del reconocimiento a nivel global teniendo en cuenta las luchas cotidianas de los pobres del mundo, que él pone en primer plano como respuesta crítica a las interacciones globales contemporáneas (Hrubec 2013).

Aunque el régimen internacional de derechos humanos garantiza formalmente los derechos humanos fundamentales de las personas migrantes, debido a su condición de vulnerabilidad como migrantes, su acceso a los derechos formalmente garantizados se ve gravemente restringido en la práctica. Las contradicciones internas entre los derechos humanos universales y la soberanía territorial, que Seyla Benhabib analiza (2006), provocan un reconocimiento erróneo estructural de los migrantes. Como nos recuerda Honneth, la denegación sistemática de derechos no sólo restringe la autonomía individual, sino que también perjudica gravemente la imagen que se tiene de sí mismo como individuo moralmente igual. Sin embargo, el contexto social de la migración viola las expectativas de reconocimiento de los migrantes no sólo en términos de igual dignidad y derechos, sino también en términos de reconocimiento social de los rendimientos individuales y la contribución a la sociedad. De esta manera, la migración socava el respeto de sí mismo y la autoestima de los individuos.

Además, la migración puede perturbar a las familias y las relaciones íntimas cuando la distancia espacial crea una distancia psicológica. En consecuencia, muchas personas migrantes tienen que reconstruir sus redes sociales y encontrar nuevas fuentes de confianza en sí mismas. Los migrantes están expuestos a un reconocimiento erróneo que afecta gravemente a su bienestar material y psicosocial. Sus experiencias de este reconocimiento erróneo son individuales pero, al mismo tiempo, por ser miembros de un grupo estructural, son estructuralmente generalizables y pueden dar lugar a reclamaciones de justicia colectivas. En sus luchas cotidianas contra el reconocimiento erróneo, los migrantes y expresan su postura crítica no sólo por la falta de reconocimiento de su dignidad en términos de igualdad, sino también por su duro trabajo y sus resultados. Esto es lo que yo entiendo por "crítica vivida”. Mediante esta crítica, los migrantes expresan sus reivindicaciones y cuestionan la legitimidad de los marcos de referencia centrados en el estado-nación para las políticas migratorias y el sistema económico global existente, reivindicando la ampliación del alcance del reconocimiento más allá del estado-nación.

Gran parte de la atención que se presta a la violación de los derechos de los migrantes se refiere a la situación de los migrantes indocumentados, un grupo de personas que cruzan las fronteras sin la documentación necesaria o que entran legalmente en un país pero que posteriormente permanecen en él sin permiso de 
residencia. Si bien es cierto que los migrantes indocumentados son más vulnerables a la exclusión, la explotación y la denegación de sus derechos, la violación de los derechos de los migrantes y el reconocimiento erróneo estructural conciernen tanto a los migrantes indocumentadas como a las documentadas. El denominador común de la experiencia vivida por los migrantes es la percepción de una falta de reconocimiento intersubjetivo y una lucha constante por reconstruir sus vidas, el desarrollo de los proyectos vitales y la autorrealización personal ${ }^{6}$. La denegación sistemática de sus derechos y la experiencia de ser tratados como miembros de segunda clase de la sociedad perjudican la autopercepción positiva de los migrantes. Aunque intuitivamente esperan igualdad de respeto y derechos junto con el reconocimiento de su contribución social y económica, experimentan un reconocimiento erróneo frente a los burócratas, empleadores y otras personas, que perciben como la encarnación del régimen regulador de la sociedad dominante.

Los relatos de los migrantes revelan diferentes ejemplos de maltrato como personas de segunda clase. Entre ellos figuran el hecho de no poder acceder más que a una gama limitada de empleos precarios o incluso ilegales, el hecho de que se les niegue un contrato de trabajo con la seguridad social básica, el hecho de no poder hacer valer sus derechos, el hecho de estar expuestos a observaciones xenófobas de los burócratas, el hecho de enfrentarse a la desconfianza y la sospecha de robo por parte de sus empleadores sobre la base de estereotipos culturales (Uhde 2014). En sus relatos, los migrantes reflejan con fuerza su vulnerabilidad a la subordinación y explotación. Incluso para acceder a los derechos, que están formalmente garantizados pero que en realidad se les niegan, se ven obligados a emplear estrategias para eludir el sistema legal. Las experiencias vividas por esos migrantes exponen cuán profundamente afectan al respeto de sí mismo y a la autoestima de un individuo la denegación de derechos y el reconocimiento de su esfuerzo laboral, y cuánto tienen que rebajar sus expectativas para mantener cierta integridad personal básica ${ }^{7}$. Esta disminución de las expectativas es lo que define el impacto de un reconocimiento erróneo estructural.

\footnotetext{
${ }^{6}$ Baso mi conceptualización de las experiencias vividas por las personas migrantes en mi trabajo anterior (Uhde 2014), así como en otros estudios basados en las narrativas de las migrantes (por ejemplo, Anderson 2000; Choudry y Hlatshwayo 2015; Hondagneu-Sotelo 2001; Parreñas 2001; Pérez y Stallaert 2016). También se basa en la experiencia de las migrantes marginalizadas hacia los países globalmente más ricos de las regiones económicas y culturales europeas y angloamericanas.

7 En mi análisis de los relatos de las personas migrantes sociales y económicos en la República Checa, identifiqué varias estrategias en las que las migrantes replantean la falta de reconocimiento social a fin de atribuir un significado positivo a su trabajo y renovar las fuentes de su autoestima. Por ejemplo, hablan de la satisfacción positiva y el reconocimiento percibido por su esfuerzo o rendimiento en momentos que deberían darse por sentados en el contexto del estado de derecho (rule of law): por ejemplo, cuando los empleadores los invitan a firmar un contrato de trabajo o les expresan su confianza (Uhde 2014).
} 
Los migrantes perciben el reconocimiento erróneo como un desfase entre sus reivindicaciones derivadas de su arduo trabajo en el país de destino, así como la falta de oportunidades para permanecer en su país de origen, por una parte, y las prácticas de discriminación y marginación, por otra. Les resulta difícil o imposible justificar las desigualdades sociales derivadas de la distinción existente entre ciudadanos y no ciudadanos. A menudo, basan sus reivindicaciones de derechos y de igual dignidad en la lógica del mérito, del trabajo duro y su contribución económica. Muchos perciben sus elecciones de migrar como inevitables, en lugar de preferibles, impuestas por circunstancias externas que no pueden controlar (Sciurba 2019). En muchos casos, tienen la intuición crítica de que esas circunstancias ni siquiera están bajo el control de sus gobiernos nacionales. Las experiencias vividas por las migrantes están llenas de testimonios de las duras condiciones económicas y sociales en el hogar, las privaciones materiales, la violencia, la impotencia y la preocupación por el futuro de sus hijos. En este contexto, la decisión de migrar no es una decisión individual, sino que se toma en el seno de la familia bajo la presión de las limitaciones estructurales.

Como he sostenido hasta ahora, la acción cotidiana de los migrantes -desde su decisión de migrar, la manipulación de los recursos disponibles y las opciones legales hasta su distanciamiento crítico de ser tratados como personas de segunda clase- es una expresión de su crítica al sistema capitalista global y al marco institucional centrado en el estado-nación en el que están atrapados. La migración es una forma de protesta contra el reconocimiento erróneo y la ausencia del derecho a no migrar (derecho al desarrollo), pero es un proceso que continúa a lo largo de sus vidas, ya que siguen siendo etiquetados como migrantes con todas las consecuencias económicas, políticas y culturales. Su crítica vivida apunta a la visión de las comunidades fronterizas que contradice la experiencia vivida de sus luchas sociales contra las circunstancias, transnacionales en su alcance, que les obligan a marcharse y la falta de instituciones transnacionales a las que puedan reclamar efectivamente sus derechos. Aunque sus luchas no adoptan la forma de la protesta política tradicional, los y las migrantes desarrollan estrategias cotidianas para navegar por el sistema, expresando así su lucha contra el reconocimiento erróneo. En el plano de la crítica vivida, también podemos identificar reivindicaciones de género que son en gran medida invisibles dentro de la protesta política organizada debido a que las estructuras de género marginan las voces de las mujeres. Sin embargo, a menudo se pasa por alto la crítica vivida por las migrantes fuera de las colectividades políticas organizadas y la acción violenta colectiva. Ariadna Estévez (2014), por ejemplo, identifica las luchas de los migrantes por el reconocimiento sólo en acciones violentas y conflictos colectivos. Pero hacer caso omiso de la 
crítica vivida por los migrantes significa pasar por alto una parte importante de la protesta social.

Sin embargo, la teoría crítica debe distinguir entre las reivindicaciones justificadas y las injustificadas porque no todas las expectativas de los agentes sociales están necesariamente justificadas. De hecho, algunas de esas expectativas se basan en prejuicios xenófobos, racistas o sexistas, de modo que sus reivindicaciones se articulan en oposición a otros grupos que quedarían excluidos y a los que se les negaría el reconocimiento. Las reivindicaciones de los actores marginalizados también pueden ser utilizadas indebidamente por otros grupos y desviadas por ellos para promover sus propios intereses o fomentar formas de exclusión o violencia. En el contexto de un reconocimiento erróneo estructural, los grupos de personas migrantes marginalizadas pueden ser manipulados por los medios de comunicación contra otros grupos de migrantes mediante falsas premisas de inclusión y reconocimiento con la condición de excluir a otros. Por consiguiente, la crítica de la injusticia vivida por las personas migrantes y sus reivindicaciones normativas deben relacionarse con el desarrollo histórico de las normas institucionalizadas de justicia y considerarse en términos de cómo extienden la validez de esas normas (Uhde 2014).

\section{Reivindicaciones de cuidado para la justicia global}

LA TEORÍA SOCIAL CRÍTICA cosmopolita necesita un sujeto de justicia adecuadamente definido. Mientras que el cosmopolitismo liberal establece a los individuos como unidad de análisis, las teorías sociales de la justicia global presuponen predominantemente colectividades organizadas. Sin embargo, al restringir la comprensión de los agentes sociales a las colectividades más o menos organizadas y a la protesta política tradicional se deja fuera a un gran grupo de personas marginalizadas. Esta deficiencia también tiene una importante repercusión en la conceptualización de las reivindicaciones de género para la justicia global.

Alison Jaggar (2014) sostiene que las teorías de la justicia global deberían prestar más atención a la investigación feminista porque la injusticia global por motivos de género es el resultado de los vínculos entre las injusticias estructurales en diversos niveles, desde el nacional y el translocal hasta el transnacional y el global, que deben formar parte de toda articulación normativa de la justicia global. Su marco conceptual de los ciclos transnacionales de vulnerabilidad de género amplía la comprensión de Young de las estructuras de género y la injusticia estructural, y revela no sólo los vínculos causales entre los diferentes aspectos y niveles de justicia, sino también su conexión con las estructuras del capitalismo global (Jaggar 2014). Jaggar sostiene 
que los análisis feministas han ampliado el concepto de ámbitos de la justicia para incluir a los hogares y las familias. De esta manera, podemos incluir lo personal en las esferas de justicia y convertirlo en una esfera de justicia global. También han ampliado el tema de la justicia para incluir a las colectividades transnacionales como agentes de las reivindicaciones de la justicia global más allá de los individuos o los estados nacionales. Y, por último aunque no menos importante, han ampliado la conceptualización del objeto de la justicia incluyendo no sólo los derechos y responsabilidades políticas, los derechos sociales y económicos y los derechos culturales, sino también la responsabilidad de las tareas domésticas y el cuidado a nivel transnacional (Jaggar 2014: 9-13). Jaggar afirma que tener en cuenta estos aspectos es un desafío para la teoría contemporánea de la justicia global.

Sin embargo, la comprensión de Jaggar del tema de la justicia parece ser incompleta. Se refiere al trabajo de Hye-Ryoung Kang (2014), que propone un enfoque de la justicia global basado en las colectividades transnacionales como sujetos de las reivindicaciones de justicia. Esto supone la existencia de un sujeto político organizado, ya que entiende las colectividades transnacionales como grupos constituidos sobre la base de organizaciones voluntarias reunidas por vulnerabilidades compartidas dentro de estructuras sociales, como las redes transnacionales de defensa de derechos. En el mismo sentido, Beck (2009) conceptualiza la subpolítica y Estévez (2014) ve las luchas por el reconocimiento de las personas migrantes sólo en una acción colectiva. Por una parte, Kang rechaza una posición nacionalista que se basa en los fundamentos comunitarios nacionales y el concepto de la nación como la comunidad más grande. Por otra parte, define su enfoque en oposición al cosmopolitismo liberal, que se basa en los fundamentos individualistas. El problema es que ella equipara erróneamente el cosmopolitismo sólo con su tradición liberal, dejando de lado la teoría crítica cosmopolita que no sólo es compatible con su concepto de sujeto transnacional colectivo de justicia, sino que también lo amplía.

Restringir la atención a las colectividades transnacionales políticamente organizadas como agentes de las reivindicaciones de justicia global determina qué tipo de reivindicaciones se ponen en primer plano y cómo se interpretan. Sin embargo, como nos recuerda Jaggar (2014), llamar la atención sobre las responsabilidades del cuidado como un aspecto relevante de la justicia es un importante logro de la teoría feminista. Las estructuras de la división del trabajo en función del género y la distribución de la responsabilidad del cuidado de los niños y niñas, el hogar y las personas mayores han ocupado durante mucho tiempo el centro de la crítica feminista de las desigualdades de género en la sociedad, que también ha considerado sus interacciones globales (Ehrenreich y Hochschild 2002; Jaggar 2014; Mahon y Robinson 2011; Zimmerman, Litt y Bose 2006). En el contexto del capitalismo 
global, con el aumento de las desigualdades globales, los riesgos globales y la migración, el cuidado se ha convertido en una cuestión de justicia global.

El sistema capitalista global se caracteriza por una intensiva ampliación (Robinson 2004, 2014) que genera cada vez más beneficios a través de la mercantilización de la vida social. Este contexto ha llevado al establecimiento de un sector precario de cuidados, mal remunerado, que se caracteriza por un creciente empleo de migrantes (Ehrenreich y Hochschild 2002; Mahon y Robinson 2011). Sin embargo, el proceso de mercantilización no ha convertido el cuidado en una cuestión pública; el cuidado sigue siendo privado y opera dentro de la economía privada. Me refiero a estos cambios en la forma social del cuidado que se está volviendo dependiente de la migración como prácticas de cuidado transnacional (Uhde 2016a), unas prácticas que entran en una relación dialéctica con la ampliación intensiva del capitalismo global y las estructuras de división del trabajo en función del género. Aunque no todos las personas trabajadoras en el sector de los cuidados son necesariamente migrantes, las trabajadoras migrantes en el sector de los cuidados como pueden considerarse un ejemplo paradigmático de las prácticas transnacionales de cuidados.

Las prácticas transnacionales de prestación de cuidados muestran cómo la economía política de la reproducción social depende de la preservación de las actuales desigualdades globales que están suministrando la mano de obra barata de las trabajadores migrantes y, al mismo tiempo, de la reproducción de las estructuras de la división tradicional del trabajo basada en el género, en la que la mayoría de los hombres no tienen que asumir la responsabilidad de la prestación de cuidados y éstos siguen siendo responsabilidad privada de las mujeres. De hecho, la perspectiva del nacionalismo metodológico crea una ilusión de igualdad entre los géneros en los países comparativamente más ricos a expensas de los países más pobres y de las personas migrantes mediante la explotación de las desigualdades globales. Resulta interesante que el cierre de las fronteras como medidas antipandémicas en marzo de 2020 haya arrojado luz sobre la fuerte dependencia de los países ricos de las prácticas de cuidados transfronterizas como piedra angular de la economía política transnacional de la reproducción social que presupone a la vez que niega la realidad de las vidas transnacionales de las trabajadoras migrantes en el sector de los cuidados.

Los estados receptores dependen de mecanismos eficaces para utilizar la mano de obra barata para el cuidado de las personas migrantes, predominantemente mujeres; sin embargo, descuidan sistemáticamente las necesidades sociales y de cuidado de las mujeres migrantes y sus responsabilidades de cuidado transnacional (Kofman y Raghuram 2015). Mediante diferentes mecanismos, los estados operan una sutil exclusión de las trabajadoras migrantes en el sector de los cuidados de la protección de los derechos laborales y sociales. A nivel global, las trabajadoras domésticas 
migrantes son los que se encuentran en la posición más vulnerable entre los trabajadores migrantes. La condición precaria (y en muchos casos indocumentada) de las trabajadoras domésticas creada por la legislaciones estatales no se utiliza para excluirlos, sino para incluirlos en condiciones desiguales (De Genova 2002). El sistema se basa de forma efectiva en una explicación nacionalista que da prioridad al interés de los ciudadanos de más edad y sus familias, a menudo a expensas de las trabajadoras domésticas migrantes a las que se les niega el reconocimiento de su igual dignidad. Adoptando la perspectiva analítica del cosmopolitismo metodológico, podemos identificar estas injusticias y al mismo tiempo reconocer las complejidades de las relaciones sociales. La migración permite a las personas mejorar algunos aspectos de sus vidas, al tiempo que las confronta con la explotación, la opresión y otras formas de injusticia estructural. Pasar por alto las discrepancias transnacionales en la condición de las personas migrantes -la diferencia entre el cambio de condición en el país de origen y el cambio de condición en el país de destino- es uno de los errores sistemáticos del nacionalismo metodológico (Beck y Beck-Gernsheim 2013).

La mayoría de las mujeres migrantes marginadas experimentan un círculo vicioso ineludible en el que su posición en las estructuras de género y su condición de migrantes institucionalizadas determinan sus oportunidades de empleo, que a menudo se limitan a trabajos estereotipados de género y de servicio (trabajo doméstico, cuidado del hogar, limpieza u otras ocupaciones elementales). Por una parte, la migración les permite escapar de ciertas expectativas de género en su país de origen y obtener un ingreso que allí no podrían obtener. Por otra parte, sin embargo, se encuentran en una posición desigual definida por el género como mano de obra de segunda clase, ya que los servicios domésticos contratados -incluso en los casos en que están formalizado ${ }^{8}$ - suelen estar excluido de muchos derechos laborales que se consideran estándar para otros sectores (por ejemplo, horarios máximos de trabajo, pago de horas extras, licencias pagadas, pago por enfermedad), y están sujetas a una serie de prácticas de explotación o expuestas al riesgo de violencia (Anderson 2000; Ehrenreich y Hochschild 2002; Parreñas 2001; Zimmerman, Litt y Bose 2006). Los países de altos ingresos o comparativamente más ricos se han beneficiado desproporcionadamente de esta situación. En este caso, las fronteras entre los estados nacionales reproducen y profundizan las desigualdades entre dos mundos sociales, haciendo que las fronteras entre ellos sean físicamente permeables (al menos en cierta medida) pero sean insuperables en sentido simbólico y material. Las fronteras

\footnotetext{
${ }^{8}$ Por ejemplo, en Austria la condición formal predominante de las trabajadoras internas es el empleo autónomo al que no se aplican los derechos laborales (Aulenbacher y Leiblfinder 2019). Además, el Convenio de la OIT sobre el trabajo de las trabajadoras domésticas no se aplica en estas situaciones.
} 
son permeables para la mercantilización de los cuidados e impenetrables para el reconocimiento social. Los derechos de las trabajadoras domésticas surgieron en la arena política internacional con una exitosa campaña a favor del Convenio de la OIT sobre el trabajo doméstico, organizada por lo que hoy es la Federación Internacional de Trabajadoras Domésticas y apoyada por otras ONG transnacionales. El Convenio sobre las trabajadoras y los trabajadores domésticos (C189) fue aprobado en junio de 2011 y establece normas internacionales para la protección de dicho tipo de trabajadores (OIT 2011). Si bien constituyó un logro impresionante, también redujo las reivindicaciones globales de cuidados a las reclamaciones de profesionalización del trabajo doméstico y el reconocimiento de los cuidados como cualquier otro trabajo ${ }^{9}$. Esto no aborda las causas estructurales del reconocimiento erróneo de los cuidados y, a largo plazo, reproduce tanto las desigualdades globales en las que se basa la economía política transnacional de la reproducción social como las vulnerabilidades estructurales transnacionales basadas en el género. Sin embargo, en las luchas cotidianas de las migrantes se articulan reivindicaciones más complejas.

Las reivindicaciones de profesionalización del trabajo doméstico presuponen la introducción de contratos despersonalizados en los hogares privados, lo que garantizaría la formalización de la institución del trabajo doméstico como empleo. Sin embargo, esto supone una dinámica internamente contradictoria. Tanto los empleadores como las trabajadoras domésticas tratan de conciliar esas tendencias contradictorias, aunque de diferentes maneras. Varios estudios muestran que las trabajadoras domésticas no reclaman principalmente la profesionalización (Ally 2009; Hondagneu-Sotelo 2001; Parreñas 2001; Pérez y Stallaert 2016; Ray y Qayum 2009). La profesionalización basada en la valoración instrumental no se corresponde plenamente con las expectativas morales de las trabajadoras domésticas. La discrepancia de las relaciones impersonales en el espacio personal del hogar hace que la valoración instrumental de sus rendimientos sea una forma de reconocimiento erróneo de su subjetividad individual. Las trabajadoras domésticas migrantes esperan el reconocimiento social tanto de sus rendimientos laborales como de su relación "de carińo" con las personas a las que cuidan, que no puede alcanzarse mediante el mero reconocimiento de los cuidados como cualquier otro trabajo. Además, Iris M. Young señala los límites del principio de rendimiento cuando se trata de reconocer a quienes prestan cuidados, pero también cuestiona la división entre el reconocimiento y la estima afectiva, que según ella se basa en el género. Young (2007: 210) argumenta que "para estimar adecuadamente a quienes prestan

\footnotetext{
9 Otro problema es que el convenio sólo tiene una fuerza legal limitada para las trabajadoras domésticas indocumentadas o las trabajadoras autónomas, que es una situación frecuente en muchos estados de la UE.
} 
cuidados es necesario separar la estima del principio de rendimiento". Las trabajadoras domésticas migrantes esperan estas dos formas distintas de reconocimiento: el reconocimiento de su duro trabajo y el reconocimiento afectivo como personas cuya identidad va más allá del trabajo doméstico. El reconocimiento afectivo es una condición importante para que hagan de su empleo una fuente de autoestima (Uhde 2016b). Esas reivindicaciones de redefinición de la estima pueden poseer un potencial normativo no realizado, que Honneth (2003) atribuye a las luchas sociales por redefinir y ampliar los principios de reconocimiento.

Si consideramos a las trabajadoras domésticas migrantes como un grupo estructural, podemos identificar reivindicaciones de reconocimiento del cuidado más allá del principio de rendimiento y las normas de mercado que dominan la sociedad capitalista (es decir, más allá de considerar el cuidado como una actividad comercial impersonal medida por la eficiencia y el beneficio), así como más allá de las relaciones primarias de carińo y amistad (es decir, más allá de considerar el cuidado como un asunto privado). Las trabajadoras domésticas migrantes también se oponen a las formas estereotipadas que limitan sus oportunidades de trabajo a la prestación de cuidados y al servicio. Además, la contradicción de cuidar a niños, niñas y personas mayores mientras no se les proporciona cuidados para ellas mismas, sus propios hijos y demás parientes en el país de origen da lugar a su crítica vivida de la justicia definida por el estado-nación y la territorialización del bienestar y la seguridad social. Así pues, la crítica vivida de las trabajadoras domésticas migrantes implica reivindicaciones más exigentes que la profesionalización del trabajo doméstico contratado. Para articular seriamente sus reivindicaciones es necesario incluir la reproducción social transnacional en la agenda de la justicia global que ponga remedio a las desigualdades basadas tanto en el género como en la nacionalidad. Además, también implica reivindicaciones de redefinición de la estima más allá de la valoración instrumentalizada de los resultados.

\section{Conclusión}

LAS PRÁCTICAS DE MIGRACIÓN TRANSNACIONAL son un reflejo de las formas en que los planes de vida de las personas se ven afectados por las interacciones globales. Aunque las personas migrantes no necesariamente interiorizan la conciencia cosmopolita, a través de sus acciones cotidianas expresan una crítica de las políticas migratorias en el marco definido por el estado-nación que los tratan como personas de segunda clase y les dificulta el acceso incluso a aquellos derechos que están formalmente garantizados. A través de sus luchas contra el reconocimiento erróneo, 
expresan sus reivindicaciones de ser miembros de pleno derecho de una comunidad cosmopolita. Las prácticas de migración transnacional y la transnacionalización de las formas de vida llevan consigo una fuente potencial de conciencia cosmopolita, aunque la realización de este potencial es sólo una posibilidad.

La migración es una decisión activa, un acto de crítica, que a menudo se considera una necesidad más que una opción preferida, ya que se toma bajo las limitaciones de las condiciones institucionalizadas del capitalismo global. Estas estructuras limitan sus acciones, pero también se transforman por ellas. Los migrantes transnacionales tienen una intuición crítica de que las estructuras económicas transnacionales y globales son fuentes de la injusticia y del reconocimiento erróneo que experimentan. Sugiero que la crítica vivida por las migrantes pone en tela de juicio la legitimidad del capitalismo global y del marco institucional y jurídico definido por el estado-nación, amplía la validez de los principios de reconocimiento y representa una articulación de las reivindicaciones normativas de un arreglo cosmopolita que podría convertirse en el importante paso hacia la justicia global. Si bien sus luchas cotidianas son aparentemente sólo individuales, si entendemos a los migrantes marginalizados como un grupo estructural, la teoría social crítica cosmopolita permite captar el rasgo colectivo de esas luchas y sus reivindicaciones generalizables de justicia.

Sin embargo, la crítica vivida por las personas migrantes suele ser ignoradas, ya que no se manifiestan en la forma tradicional de protesta política. Pasar por alto la crítica vivida por los migrantes lleva a ignorar esas expectativas morales y sus reivindicaciones que no están incluidas en la agenda de las colectividades transnacionales organizadas. Argumento que a través de su crítica vivida del reconocimiento erróneo, las trabajadoras domésticas expresan reivindicaciones que van más allá de las demandas de reconocimiento del cuidado como trabajo de acuerdo con el principio del rendimiento, que es la principal demanda de las colectividades organizadas de trabajadores y trabajadoras migrantes domésticos. Las reivindicaciones de las trabajadoras domésticas migrantes implican demandas más ambiciosas de justicia global de remediar no sólo las causas estructurales de la injusticia que se derivan del capitalismo global y del marco institucional y jurídico definido por los estadosnacionales, sino también transformar las estructuras de género como sistema de atribución de valor social. 


\section{Referencias Bibliográficas}

Ally, Shireen. 2009. From Servants to Workers. Ithaca: Cornell U.P.

Anderson, Bridget. 2000. Doing the Dirty Work. The Global Politics of Domestic Labor. Londres \& Nueva York: Zed Books.

Aulenbacher, Brigitte y Michael Leiblfinger. 2019. The "fictitious commodity" care and the reciprocity of caring. En Capitalism in Transformation, eds. R. Atzmüller et al., 245-260. Edward Elgar Publishing.

Beck, Ulrich. 2006. Cosmopolitan Vision. Cambridge: Polity Press.

Beck, Ulrich. 2009. World at Risk. Cambridge: Polity Press.

Beck, Ulrich y Elisabeth Beck-Gernsheim. 2013. Distant Love. Cambridge: Polity Press.

Beck, Ulrich y Natan Sznaider. 2006. Unpacking cosmopolitanism for the social sciences The British Journal of Sociology 57 (1): 1-23.

Benhabib, Sheyla. 2006. Another Cosmopolitanism. Oxford: Oxford U.P.

Brettell, Caroline B. y James F. Hollifield. 2000. Migration Theory. Talking Across Disciplines. Nueva York \& Londres: Routledge.

Castles, Stephen. 2010. Understanding Global Migration. Journal of Ethnic and Migration Studies 36 (10): 1565-1586.

Castles, Stephan, Ozkul, Cubas Derya y Magdalena Arias. (eds.). 2015. Social Transformation and Migration. Nueva York: Palgrave.

Choudry, Aziz y Mondli Hlatshwayo (eds). 2015. Just Work? Migrant Workers' Struggles Today. Londres: Pluto Press.

Delgado Wise, Raúl. 2018. Is there a space for counterhegemonic participation? Civil society in the global governance of migration. Globalization 15 (6): 746-761.

De Genova, Nicholas P. 2002. Migrant "illegality" and deportability in everyday life. Annual Review Anthropology 31: 419-447.

De Genova, Nicholas. 2018. Anonymous Black and Brown Bodies. Praga: The Czech Academy of Science, 30 May 2018. Accesible: http://globalnikonflikty. cz/archives/1382. 
Delanty, Gerard. 2009. The Cosmopolitan Imagination. The Renewal of Critical Social Theory. Cambridge: Cambridge U.P.

Ehrenreich, Barbara y Arlie R. Hochschild (eds.). 2002. Global Woman. Nannies, Maids, and Sex Workers in the New Economy. Nueva York: A Metropolitan/ OWL Book.

Estévez, Ariadna. 2014. Derechos humanos, migración y conflicto. México: UNAM-CISAM.

Fine, Robert. 2007. Cosmopolitanism. Londres: Routledge.

Glick Schiller, Nina. 2009. A Global Perspective on Transnational Migration: Theorizing Migration without Methodological Nationalism. Centre on Migration, Policy and Society, Working Paper No. 67. Oxford: University of Oxford, COMPAS.

Hondagneu-Sotelo, Pierrette. 2001. Doméstica. Immigrant Workers Cleaning and Caring in the Shadows of Affluence. Berkeley, Los Angeles, Londres: University of California Press.

Honneth, Axel. 2006. Redistribución como reconocimiento. Una respuesta a Nancy Fraser. En ¿ Redistribución o reconocimiento? Nancy Fraser y Axel Honneth, 89-148. Madrid: Morata.

Hrubec, Marek. 2012. Authoritarian versus Critical Theory. International Critical Thought 2 (4): 431-444.

Hrubec, Marek. 2013. An Articulation of Extra-Territorial Recognition. En Global Justice and the Politics of Recognition, ed. Tony Burns, 271-295. Nueva York: Palgrave.

ILO. 2011. Convention Concerning Decent Work for Domestic Workers. http:// www.ilo.org/wcmsp5/groups/public/@ed_norm/@relconf/documents/meetingdocument/wcms_157836.pdf. Accessed 4 December 2016.

Ingram, James D. 2013. Radical Cosmopolitics. The Ethics and Politics of Democratic Universalism. Nueva York: Columbia U.P.

Jaggar, Alison M. (ed.). 2014. Gender and Global Justice. Cambridge: Polity Press.

Kang, Hye-Ryoung. 2014. Transnational Women's Collectivities and Global Justice. En Gender and Global Justice, ed. A. M. Jaggar, 40-61. Cambridge: Polity Press. 
King, Russell. 2012. Theories and Typologies of Migration? Willy Brandt Series of Working Papers in International Migration and Ethnic Relations 3/12. Malmö University.

Kofman, Eleonore y Parvati Raghuram. 2015. Gendered migrations and global social reproduction. Basingstoke: Palgrave Macmillan.

Mahon, Rianne y Fiona Robinson (eds.). 2011. Feminist ethics and social policy. Vancouver, Toronto: UBC Press.

Parreñas, Rhacel S. 2001. Servants of Globalization. Stanford: Stanford U.P.

Pérez, Inés y Christiane Stallaert. 2016. The professionalization of paid domestic work and its limits. European Journal of Women's Studies 23 (2): 155-168.

Ray, Raka y Seemin Qayum. 2009. Cultures of Servitude. Stanford: Stanford U.P.

Robinson, William. 2004. A Theory of Global Capitalism. Baltimore: The Johns Hopkins U.P.

Robinson, William. 2014. Global Capitalism and the Crisis of Humanity. Nueva York: Cambridge UP.

Sager, Alexander. 2018. Towards a Cosmopolitan Ethics of Mobility. Cham: Palgrave Macmillan.

Sciurba, Alessandra. 2019. Vulnerability, Freedom of Choice and Structural Global Injustice. En Challenging the Borders of Justice in the Age of Migration, ed. J.C. Velasco y MC. La Barbera, 225-242. Cham: Springer.

Sklair, Leslie. 2002. Globalization. Oxford: Oxford U.P.

Sklair, Leslie. 2003. The Transnational Capitalist Class. Malden \& Oxford: Blackwell Publishing.

Turégano, Isabel. 2019. Ethical Dimensions of Migrant Policies. In Challenging the Borders of Justice in the Age of Migration, ed. J.C. Velasco y MC. La Barbera, 95-116. Cham: Springer.

Uhde, Zuzana. 2014. Zneuznání v př́bězích migrace [Misrecognition in migration life stories]. In Vlastni cestou? Zivotni dráhy v pozdně moderni společnosti, ed. Hana Hašková, 285-308. Praha: SLON.

Uhde, Zuzana. 2016a. From women's struggles to distorted emancipation. International Feminist Journal of Politics 18 (3): 390-408.

Uhde, Zuzana. 2016b. Social bias within the institution of hired domestic care. Civitas - Revista de Ciências Sociais 16 (4): 684-709. 
Vertovec, Steven. 2009. Transnationalism. Nueva York: Routledge.

Waldinger, Roger D. y David Fitzgerald. 2004. Transnationalism in Question. American Journal of Sociology 109 (5): 1177-1195.

Wimmer, Andreas y Nina Glick Schiller. 2002. Methodological nationalism and beyond nation-state building, migration and the social sciences. Global Networks 2 (4): 301-334.

Young, Iris M. 2011. Responsibility for Justice. Nueva York: Oxford U.P.

Young, Iris M. 2007. Recognition of Love's Labor. En Recognition and Power: Axel Honneth and the Tradition of Critical Social Theory, ed. B. van den Brink and D Owen, 189-212. Cambridge: Cambridge U.P.

Young, Iris M. 2006. Responsibility and Global Justice. Social Philosophy \& Policy 23: 102-130.

Young, Iris M. 2005. Lived Bodies vs. Gender. En On Female Body Experience, ed. Iris M. Young, 12-26. Oxford: Oxford U.P.

Young, Iris M. 2000. Inclusion and Democracy. Oxford: Oxford U.P.

Young, Iris M. 1990. Justice and the Politics of Difference. Princeton: Princeton U.P.

Zimmerman, Mary K., Jacquelyn S. Litt, y Christine E. Bose (eds.). 2006. Global Dimensions of Gender and Care. Stanford: Stanford U.P. 
